\title{
Anti-lipopolysaccharide factor $D$ from kuruma shrimp exhibits antiviral activity
}

\author{
Hai-Shan Jiang ${ }^{1} \cdot$ Li-Xia Lv $^{1} \cdot$ Jin-Xing Wang ${ }^{1}$
}

Received: 25 December 2020 / Accepted: 17 June 2021 / Published online: 13 July 2021

(c) The Author(s) 2021

\begin{abstract}
Anti-lipopolysaccharide factors (ALFs) exhibit a potent antimicrobial activity against a broad range of bacteria, filamentous fungi, and viruses. In previous reports, seven groups of ALFs (groups A-G) were identified in penaeid shrimp. Among them, group D showed negative net charges and weak antimicrobial activity. Whether this group has antiviral function is not clear. In this study, the ALF sequences of penaeid shrimp were analyzed, and eight groups of ALF family (groups A-H) were identified. The four ALFs including MjALF-C2, MjALF-D1, MjALF-D2, and MjALF-E2 from kuruma shrimp Marsupenaeus japonicus were expressed recombinantly in Escherichia coli, and the antiviral activity was screened via injection of purified recombinant ALFs into shrimp following white spot syndrome virus (WSSV) infection. Results showed that the expression of $V p 28$ (WSSV envelope protein) decreased significantly in the MjALF-D2-injected shrimp only. Therefore, MjALF-D2 was chosen for further study. Expression pattern analysis showed that MjAlf-D2 was upregulated in shrimp challenged by WSSV. The WSSV replication was detected in RNA, genomic DNA, and protein levels using VP28 and Iel (immediate-early gene of WSSV) as indicators in MjALF-D2-injected shrimp following WSSV infection. Results showed that WSSV replication was significantly inhibited compared with that in the rTRX- or PBS-injected control groups. After knockdown of MjAlf-D2 in shrimp by RNA interference, the WSSV replication increased significantly in the shrimp. All these results suggested that MjALF-D2 has an antiviral function in shrimp immunity, and the recombinant ALF-D2 has a potential application for viral disease control in shrimp aquaculture.
\end{abstract}

Keywords Anti-lipopolysaccharide factors · Cationic and anionic antimicrobial peptides · White spot syndrome virus . Viral envelope protein

\section{Introduction}

White spot syndrome (WSS) in crustaceans is caused by WSS virus (WSSV), which is an enveloped circular doublestranded DNA virus, and it is a severely infectious disease that results in $100 \%$ mortality of shrimp within 3-10 days after infection (Peng et al. 2001). WSSV is a virulent pathogen not only to shrimp but also to wider crustacean species. Understanding the molecular mechanisms of the shrimp

Edited by Xin Yu

Hai-Shan Jiang and Li-Xia Lv contributed equally to this work.

Jin-Xing Wang

jxwang@sdu.edu.cn

1 Shandong Provincial Key Laboratory of Animal Cell and Developmental Biology, School of Life Sciences, Shandong University, Qingdao 266237, China immune response against viral infection could provide new strategies for the disease control. Therefore, several studies were carried out, and great progress was achieved with shrimp immunity, especially in the discovery of antiviral effectors (Wang et al. 2014a; Zheng et al. 2019). Among them, antimicrobial peptides (AMPs), as the effector molecules, play important roles in shrimp antiviral immunity.

Several kinds of AMPs have been identified in shrimp, including penaeidins, crustins, anti-lipopolysaccharide factors (ALFs), and stylicins (Tassanakajon et al. 2011, 2018). Most of the peptides with net-positive charges possess antibacterial activity against Gram-positive or -negative bacteria, fungi, and viruses. ALF was first reported in chelicerates (horseshoe crab, Limulus polyphemus) as a potent anticoagulant factor (Morita et al. 1985; Ohashi et al. 1984; Tanaka et al. 1982). Since then, ALFs were identified in various crustaceans, such as portunid crabs (Afsal et al. 2012), freshwater prawn (Ren et al. 2012), crayfish (Jiravanichpaisal 
et al. 2007; Sun et al. 2011) and penaeid shrimp, such as Litopenaeus vannamei, Fenneropenaeus chinensis, Penaeus monodon and M. japonicus (Tassanakajon et al. 2018). Besides a conserved signal peptide, the sequences of mature ALF peptides contain a lipopolysaccharide (LPS)-binding domain formed between two conserved cysteine residues. The three-dimensional structure of ALFs consists of three $\alpha$-helices and four stranded $\beta$-sheets (Yang et al. 2009). A central $\beta$-hairpin structure formed by 20 amino acid residues between two cysteines is stabilized by a disulfide bond. The central $\beta$-hairpin structure is the ALF functional domain which is involved in the recognition and binding of components from microbial cell walls (Schmitt et al. 2016).

The ALFs from penaeid shrimp constitute a diverse and multigenic family of AMPs. They are firstly divided into three groups (groups A-C) on the basis of sequence similarities and phylogenetic analysis (Tassanakajon et al. 2011). Group A consists of anionic and cationic polypeptides, whereas groups $\mathrm{B}$ and $\mathrm{C}$ are composed of highly cationic polypeptides. Subsequently, group D shrimp ALFs with negative net charges are identified and display impaired LPS-binding activity, and weak antimicrobial activity (Rosa et al. 2013). Then, a new group of ALFs, including MjALFE1 and -E2 (designated as group E), was identified from Marsupenaeus japonicus (Jiang et al. 2015); they exhibit antimicrobial activity against bacteria. In addition, two novel groups of the family (groups F and G) were recently identified in L. vannamei (Matos et al. 2018). Group F is composed of cationic peptides with antibacterial and antifungal activities, whereas the antimicrobial activities of group $\mathrm{G}$ belonging to anionic peptides have not been identified (Matos et al. 2018). The ALFs with net positively charged LPS-Binding Domain (such as groups B, C, and F), which is highly conserved in sequence of each group, exhibit high antimicrobial activity, and anionic ALFs (such as group D) have weak or no antimicrobial activity. The seven ALFs could be simultaneously identified in a single shrimp, such as L. vannamei and M. japonicus (Jiang et al. 2015; Matos et al. 2018), possibly suggesting that different ALFs may act synergistically to improve their antimicrobial activity in vivo.

Functional analyses showed that ALF proteins exhibit a potent antimicrobial activity against a wide range of micro-organisms, including Gram-positive and Gramnegative bacteria and filamentous fungi (Rosa et al. 2013). ALFs also play an important role in the defense against viral pathogens in crustaceans. In vivo function analysis in crayfish Pacifastacus leniusculus showed that the knockdown expression of the ALF gene by double-stranded RNA (dsRNA) injection enhanced the expression of VP28, the envelope protein of WSSV (Liu et al. 2006). Silencing the LvALF in $L$. vannamei by RNA inference could also increase mortality after WSSV infection (de la Vega et al. 2008). The synthetic LBD (LPS-binding domain) of $F c$ ALF and FcALF2 (group B) from the shrimp F. chinensis could inhibit the replication of WSSV in vivo (Li et al. 2014; Liu et al. 2005). The ALFPm3 (group C) identified in Penaeus monodon exhibits anti-WSSV function by binding to the target structural protein complex of WSSV and leading to virion damage (Methatham et al. 2017).

Previous studies identified seven groups of ALFs (groups A-G) in kuruma shrimp M. japonicus, and the antibacterial functions of some of these ALFs were studied (Jiang et al. 2015). The anionic ALF-Ds showed weak antimicrobial activity (Rosa et al. 2013). Whether the anionic ALFs also has antiviral activity is not clear. In the present study, the phylogenetic tree of ALFs from penaeid shrimp was reconstructed, and a new group of ALF family (group H) was identified. The function of anionic MjALFD2 in shrimp immunity was analyzed, and the anti-WSSV activity of ALF was authenticated in kuruma shrimp.

\section{Results}

\section{Eight groups of the ALF family were identified in penaeid shrimp}

Nine ALF members were identified in the transcriptomic sequencing of shrimp M. japonicus. Then, 29 sequences of ALFs (GenBank accession numbers listed in Fig. 1A) were collected in four other penaeid shrimp species, namely, $F$. chinensis, F. penicillatus, L. vannamei, and P. monodon, from the GenBank database for reconstruction of phylogenetic trees. The members of the ALF family in penaeid shrimp were divided into eight groups (groups A-H, Fig. 1A), and a new group (group H) was identified. These groups could be classified into five clusters, i.e., cluster I (groups C, B, and F), Cluster II (group H), cluster III (group A), cluster IV (group D), and cluster V (groups $\mathrm{E}$ and $\mathrm{G}$ ). The newly identified group $\mathrm{H}$ is composed of cationic ALFs with isoelectric points ranging from 9.14 to 9.80 present in M. japonicus, L. vannamei, and Penaeus monodon (Fig. 1B). The original MjALFE1 in the previous report (Jiang et al. 2015) was reclassified as MjALF-H in the reconstructed phylogenetic trees (Fig. 1A). The function of MjALF-E1 was studied, and the results revealed that it could strongly bind to bacteria, and has antibacterial activity (Jiang et al. 2015). The original MjALF-D1 (a cationic peptide) was reclassified as group F (MjALF-F) in the phylogenetic trees (Fig. 1A). In summary, eight groups of ALF family were identified in kuruma shrimp $M$. japonicus, and there has not been any functional study conducted for group D member MjALF-D2. 


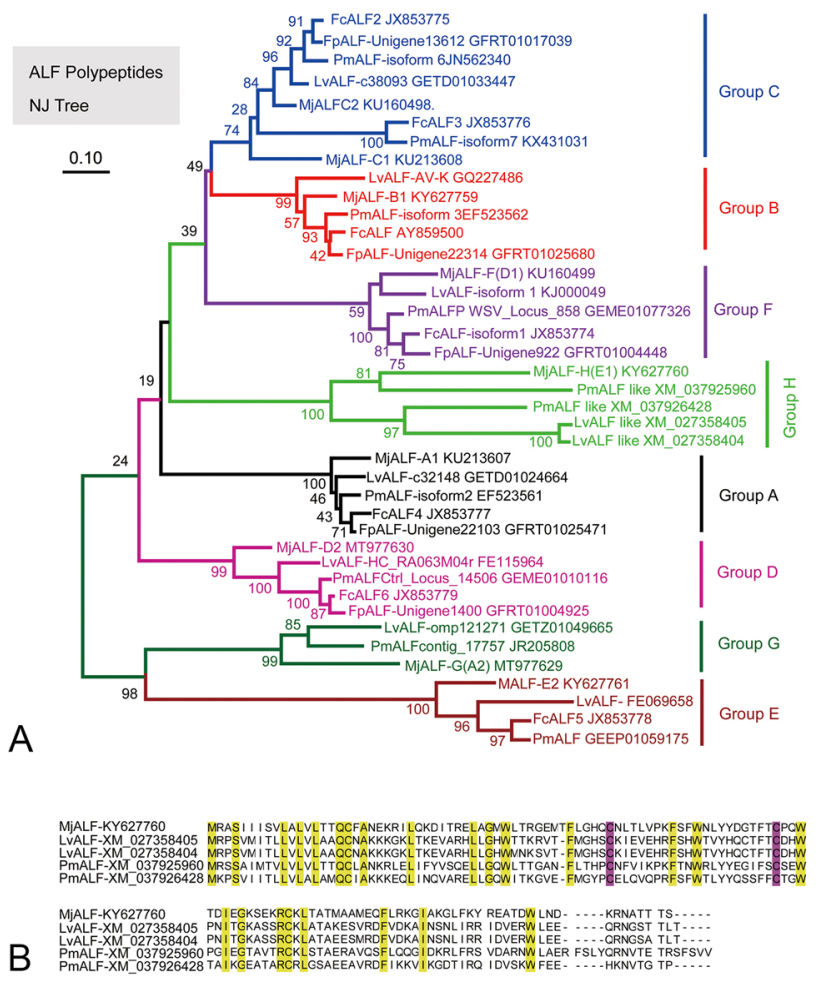

Fig. 1 Phylogenetic tree of ALFs and alignment of group ALFH. A Phylogenetic tree of ALFs from penaeid shrimp constructed via MEGA by using neighbor-joining method. Nine ALF members belong to eight groups of the ALF family identified in M. japonicus. B Alignment of amino acid sequences of five ALFs in group $\mathrm{H}$. Fc, Fenneropenaeus chinensis; Fp, Fenneropenaeus penicillatus; Lv, Litopenaeus vannamei; $\mathrm{Mj}$, Marsupenaeus japonicus; Pm, Penaeus monodon. The LPS-binding domain is located at two cysteine residues

\section{Antiviral activity screening using recombinant MjALFs}

Four recombinant ALF(rALF) polypeptides, including MjALF-C2 (GenBank accession no. KU213608), MjALFD1 (KU160499), MjALF-D2 (MT977630), and MjALF-E2 (KY627761), were successfully expressed in Escherichia coli. After the recombinant ALFs were purified via Ni-NTA chromatography (Fig. 2A), the antiviral effect of the rALFs was screened initially by injecting the rALF and WSSV mixture into shrimp; viral replication was detected at $36 \mathrm{~h}$ post injection using the expression of $V p 28$ (the envelope protein of WSSV) as the indicator. The results showed that WSSV replication in gills of the rMjALF-D2-injected shrimp was inhibited significantly compared with that in other rALFs and the controls (Fig. 2B). For further confirmation of the results, PBS and thioredoxin (TRX)-tag protein purified from the parent vector pET-32a (+) were used as controls in the antiviral activity analysis. The results showed that compared with PBS and TRX, rMjALF injection significantly

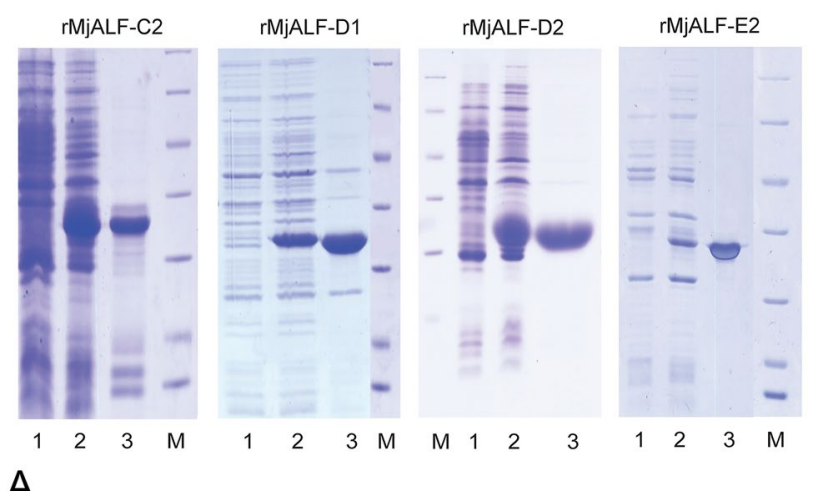

A

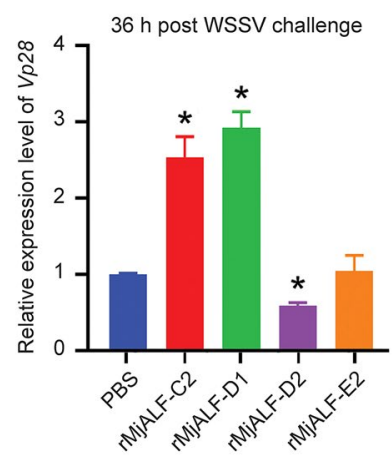

B

Fig. 2 Recombinant expression and purification of four MjALFs and antiviral activity analysis in shrimp. A Purification of MjALFs expressed in E. coli. and Lane 1, total proteins of E. coli with pET32a/Alf plasmids without induction; lane 2, total proteins of $E$. coli with pET32a/Alf after IPTG induction; lane 3, purified MjALFs; and lane M, standard protein markers. B Expression of $V p 28$ in gills of rMjALF-injected shrimp challenged by WSSV, as analyzed by qPCR using $\beta$-Actin as internal control. C $\mathrm{qPCR}$ was used to analyze the expression of $V p 28$ (encoding the envelope protein of WSSV) in hemocytes of rMjALF-D2-injected shrimp challenged by WSSV. PBS and rTRX injection were used as controls. Asterisks indicate statistical significance $(* P<0.05 ; * * P<0.01)$ analyzed by Student's $t$ test

inhibited WSSV replication in shrimp hemocytes (Fig. 2C). All the results suggested that MjALF-D2 may possess antiWSSV function in shrimp.

\section{MjAlf-D2 was detected in all tissues tested, and upregulated in shrimp challenged by WSSV}

The tissue distribution of MjAlf-D2 in the hemocytes, heart, hepatopancreas, gills, stomach, and intestine of untreated shrimp was detected by semi-quantitative reverse transcription polymerase chain reaction (RT-PCR). The results showed that MjAlf-D2 was detected in all tissues examined, and it was highly expressed in gills, stomach, and intestine (Fig. 3A). The expression patterns of MjAlf-D2 in different tissues of shrimp challenged by WSSV were analyzed by quantitative real-time PCR (qPCR), and the results showed 
Fig. 3 Tissue distribution and expression patterns of MjAlf-D2 in shrimp. A Tissue distribution of MjAlf-D2 in normal shrimp analyzed by RT-PCR. B Expression pattern of MjAlf-D2 in hemocytes of shrimp challenged by WSSV was analyzed by qPCR. C Expression pattern of MjAlf-D2 in intestine of WSSVchallenged shrimp analyzed by qPCR. D Expression pattern of MjAlf-D2 in gills of shrimp challenged by WSSV. Student's $t$ test was used to analyze statistical significances $(* P<0.05$; $* * P<0.01 ; * * * P<0.001)$

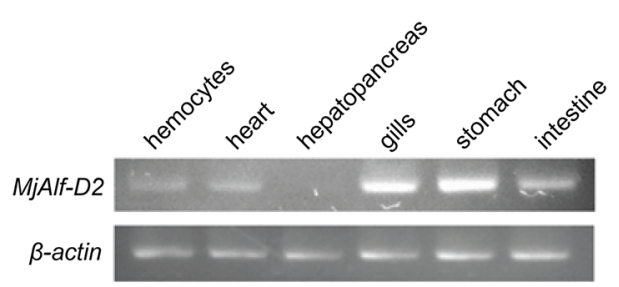

A

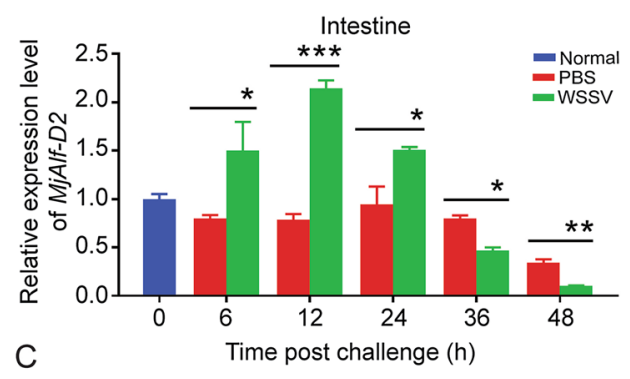

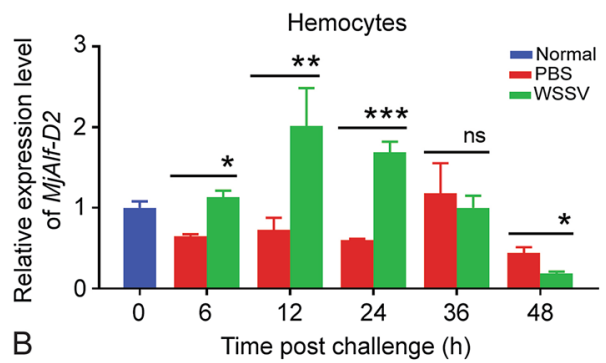

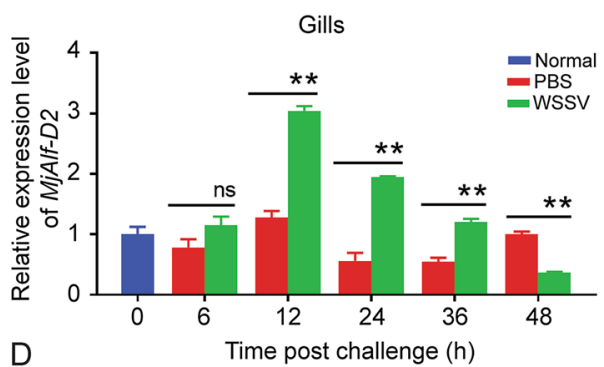

that after WSSV challenge, MjAlf-D2 was significantly upregulated in the hemocytes (Fig. 3B), intestine (Fig. 3C), and gills (Fig. 3D) of shrimp. The expression of MjAlf-D2 was upregulated from $6 \mathrm{~h}$, reached the highest level at $12 \mathrm{~h}$, and started to recover from $36 \mathrm{~h}$ post WSSV infection. These results suggested that MjALF-D2 was responsible for WSSV infection in shrimp.

\section{Recombinant MjALF-D2 inhibited the replication of WSSV in vivo}

WSSV proliferation in RNA, genomic DNA, and protein levels was detected in rMjALF-D2-injected shrimp following WSSV infection, with Vp28 and Iel (immediate-early gene of WSSV) as indicators, using qPCR to confirm the anti-WSSV activity of rMjALF-D2. The results showed that compared with PBS and TRX injection control, the rMjALFD2-injected group showed significant reduced expression of Vp28 and Iel in gills at $36 \mathrm{~h}$ (Fig. 4A) and $48 \mathrm{~h}$ (Fig. 4B) post WSSV injection. The expression of VP28 in gills of the shrimp was also detected at the protein level by Western blotting, and similar results were obtained (Fig. 4C). These results suggested that rMjALF-D2 inhibits WSSV replication in shrimp.

\section{VP28 and le1 expression increased significantly in MjALF-D2-silenced shrimp}

MjAlf-D2 RNAi was performed in vivo to further confirm the role of MjALF-D2 in shrimp antiviral immunity. MjAlf$D 2$ could be knocked down by approximately $65 \%$ by the injection of MjAlf-D2 dsRNA ( $7 \mu \mathrm{g} / \mathrm{g}$ shrimp) compared with the $d s G f p$ injected in the controls (Fig. 5A). The MjAlf$D 2$-silenced shrimp were challenged with WSSV, and the expression of $V p 28$ and $I e l$ was detected at mRNA, genomic DNA, and protein levels at 36 and $48 \mathrm{~h}$ post WSSV injection. The results showed that the expression of VP28 increased in gills of shrimp at the protein level compared with that of $d s G f p$ (Fig. 5B). The expression of Vp28 and IeI at RNA and genomic DAN levels was also analyzed by qPCR. The results showed that this expression increased significantly in the MjAlf-D2-knockdown shrimp challenged by WSSV at $36 \mathrm{~h}$ (Fig. 5C) and $48 \mathrm{~h}$ (Fig. 5D) post viral injection. All the results suggested that MjALF-D2 exerts important roles in shrimp antiviral immunity.

\section{Discussion}

In this study, the phylogenetic tree of the ALF family from penaeid shrimp was reconstructed, and a new group (group $\mathrm{H})$ was identified. Eight groups of ALF family (A-H) were found simultaneously in kuruma shrimp M. japonicus, and the antiviral activity of ALFs in the shrimp was screened using recombinant ALF polypeptides. The anionic ALF-D2 possessed anti-WSSV activity, and this inhibitory activity was further confirmed by RNAi and "overexpression" of MjALF-D2 in shrimp. The results suggested that MjALFD2 has a potential application for viral disease control in shrimp aquaculture.

Shrimp have an efficient and sophisticated innate immune system that provides protection against invading pathogens. In immune responses, shrimp recognize pathogens via germline-encoded proteins called pattern recognition receptors by sensing pathogen-associated molecular patterns from 

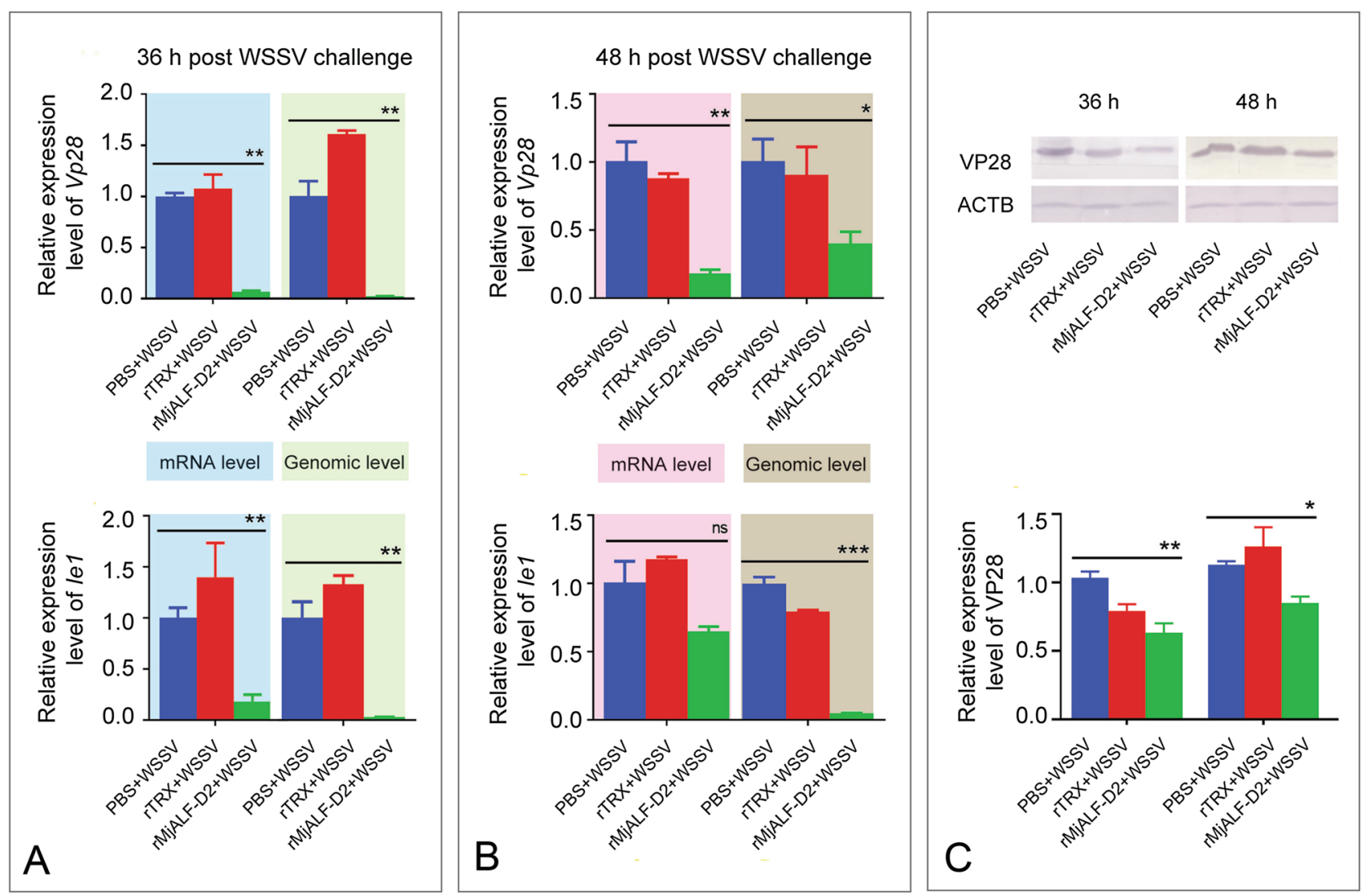

Fig. 4 Recombinant MjALF-D2 inhibits WSSV replication in shrimp. A, B Mixture of rMjALF-D2 and WSSV was injected into shrimp. Expression of $V p 28$ (upper panel) and Iel (lower panel) in mRNA and DNA levels was analyzed by qPCR at $36 \mathrm{~h}(\mathrm{~A})$ and $48 \mathrm{~h}(\mathrm{~B})$ in gills post WSSV injection. PBS and TRX-tag (rTRX) injection were used as controls. C Western blotting was used to analyze the expression of VP28 at protein level in gills of shrimp at 36 and $48 \mathrm{~h}$ after

different micro-organisms (Wang and Wang 2013). Cellular and humoral immunities are initiated in the animals against pathogens by phagocytosis, nodulation, encapsulation, melanization, extracellular traps ( $\mathrm{Ng}$ et al. 2013) (belonging to cellular responses), or by secretion of AMPs, inhibitors of proteinases, and reactive oxygen species or reactive nitrogen species (belonging to humoral responses) (Bi et al. 2015; Ng et al. 2013; Tassanakajon et al. 2018; Yang et al. 2016a). The AMPs are considered as major humoral immune effectors against invading pathogens by killing or controlling the invading micro-organisms. Among different classes of AMPs, the ALFs are the diverse family of AMP in penaeid shrimp. ALFs exhibit broad-spectrum and powerful antimicrobial activity against major shrimp pathogens. Previous studies identified seven groups of ALFs in M. japonicus (Jiang et al. 2015; Matos et al. 2018). Groups A and C were found in a broad range of tissues, including hemocytes, heart, hepatopancreas, gills, stomach, and intestine. ALFBs had a high expression level in hemocytes, heart, and
WSSV and recombinant protein infection $\beta$-Actin (ACTB) was used as loading control. The bands on the membranes of western blot were digitalized using ImageJ software based on three independent repeats (lower panel). The qPCR data were analyzed by Student's $t$ test and asterisks indicated significant difference $(* P<0.05 ; * * P<0.01$; $* * * P<0.0001)$

intestine. MjALF-D had a low expression in hemocytes, and upregulated by WSSV challenge. MjALF-E had low expression level in hepatopancreas. Group F (originally designated as MjALF-D1) was discovered in heart, gill, stomach, and intestine, and had a high expression in stomach. Group $\mathrm{G}$ (originally designated as MjALF-A2, GenBank accession no. MT977629) was mainly distributed in the stomach (Jiang et al. 2015). In the present study, group $\mathrm{H}$ was identified as a new group of the ALF family in M. japonicus, L. vannamei, and $P$. monodon. Eight groups of ALFs were present in a single shrimp, indicating that the different ALFs may act synergistically to improve their antimicrobial activities in vivo. Simultaneous exposure of an invading pathogen to multiple different AMPs may be a critical mechanism for the host effective immune response. A recent study in fruit fly using CRISPR gene editing to knock out all known inducible AMPs showed that AMPs have synergistic, additive, and highly specific microbicidal effects in Drosophila (Hanson 

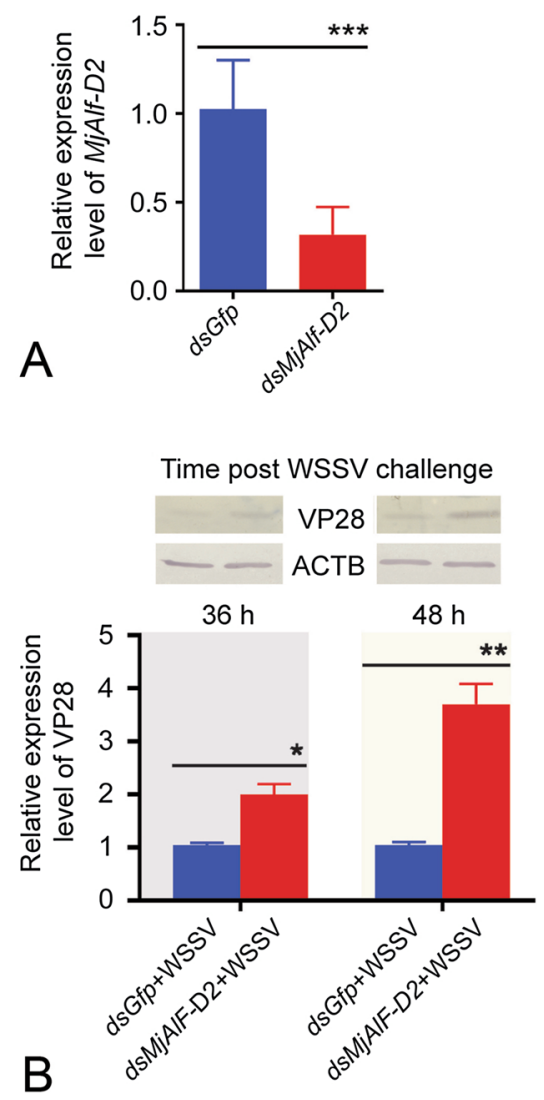
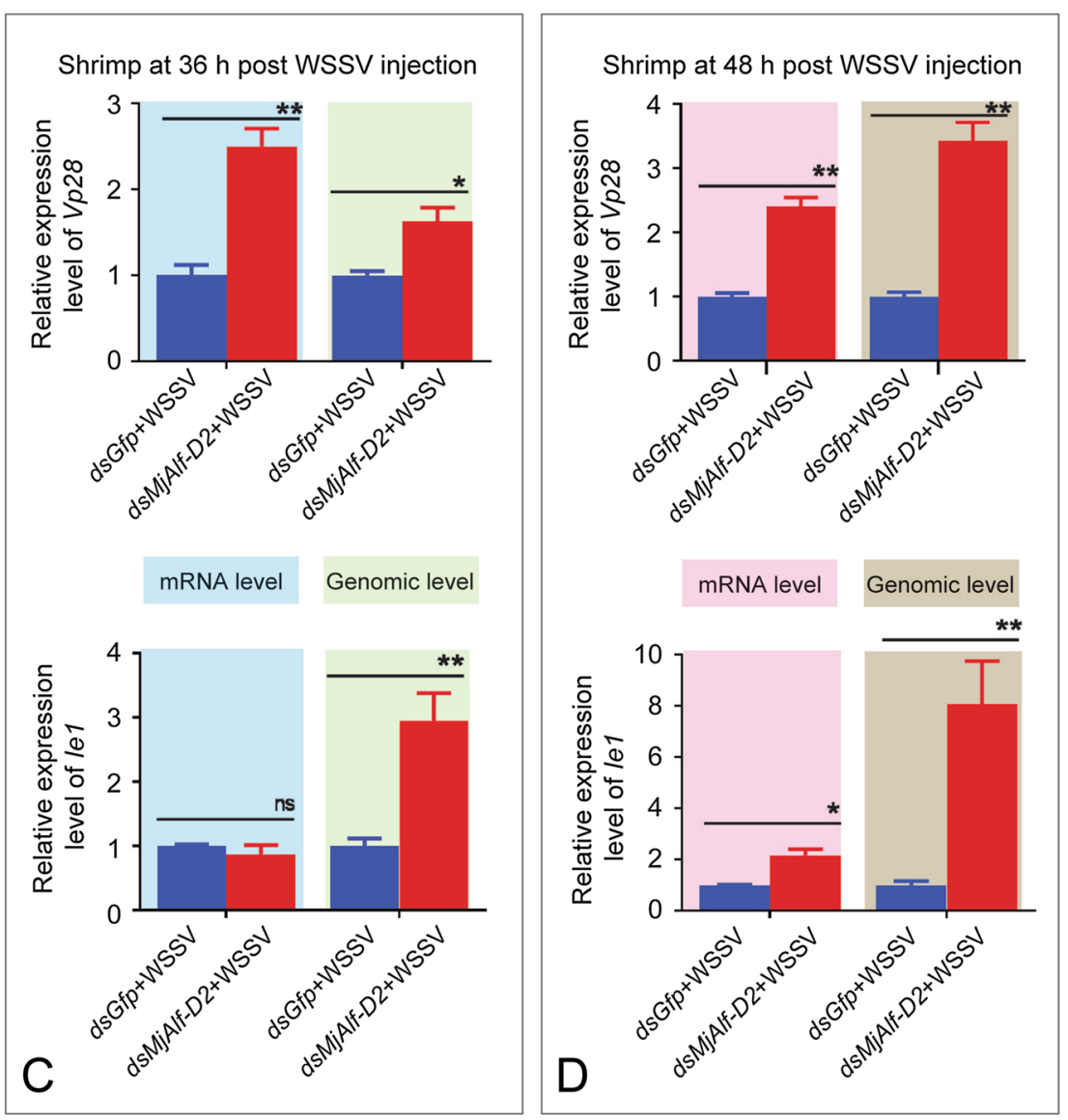

Fig. 5 Expression of $\mathrm{Vp} 28$ and Iel increased significantly in MjAlfD2-silenced shrimp. A Efficiency analysis of MjAlf-D2 RNAi in shrimp analyzed by qPCR. B Western blotting was performed to analyze the expression of VP28 in gills of MjAlf-D2-knockdown shrimp at 36 and $48 \mathrm{~h}$ post WSSV challenge. The western blot results were digitalized using ImageJ software based on three independent repeats, and shown in the lower panel of b. C Expression of $V p 28$ (upper panel) and Iel (lower panel) in mRNA and genomic DNA levels in gills of shrimp at $36 \mathrm{~h}$ post WSSV infection analyzed by qPCR. D Expression of Vp28 (upper panel) and Ie1 (lower panel) in mRNA and genomic DNA levels in gills of shrimp at $48 \mathrm{~h}$ post WSSV infection was analyzed by qPCR. Significant differences were indicated by asterisks $\left({ }^{*} P<0.05 ; * * P<0.01\right)$ analyzed by Student's $t$ test

antiviral activity of ALFs may be mediated by direct interaction with WSSV proteins. The antiviral mechanism of MjALF-D2 may be similar with that of other AMPs, i.e., it damages the virions by interaction with the viral envelope.

Shrimp aquaculture has grown rapidly and become a major global industry that contributes significantly to socioeconomic development in many countries in Asia and the Americas. However, a succession of bacterial and viral diseases affects the sustainable development of the industry. Shrimp AMPs are small peptides, which are important effectors against a wide spectrum of pathogens in the innate immune response of shrimp. Antibiotics have been used widely in aquaculture, resulting in the development and spread of resistance. Antibiotic resistance has become a global problem due to the increased multidrug-resistant pathogens along with a gradually decline in the discovery of new antibiotics for use in human and animal health 
(Mookherjee et al. 2020). AMPs are potential alternative of antibiotics as they affect a wide spectrum of pathogens, including viruses. WSSV is the most devastating virus in shrimp aquaculture. It infects all cultured penaeid shrimp species, and causes substantially economic losses globally. The potential use of ALFs in shrimp disease control has been reported previously (Supungul et al. 2017). Administering rALFPm3 could increase shrimp survival after WSSV infection. In the present study, the injection of rMjALF-D2 could inhibit WSSV replication in shrimp, and thus be beneficial to shrimp aquaculture. Therefore, shrimp ALFs have potential therapeutic uses to overcome severe viral disease outbreaks in shrimp aquaculture.

\section{Materials and methods}

\section{Immune challenge, tissue collection and total RNA extraction}

M. japonicus shrimp (8-10 g/individual) were bought from the market in Jinan, Shandong Province, China, and cultured as described previously (Niu et al. 2019). The shrimp were acclimated for $48 \mathrm{~h}$ in tanks with aerated artificial seawater (salinity between $24\left(\mathrm{w} / \mathrm{v}\right.$ ) and 26 ) at $\sim 24^{\circ} \mathrm{C}$, and randomly taken for use in the following experiments. For pathogen challenge, the shrimp were divided into two groups (30 individuals per group): one group was injected with WSSV $\left(3.2 \times 10^{5}\right.$ copies per shrimp) and the other (control group) was injected with same volume of PBS (Jiang et al. 2013). Hemolymph was collected from five shrimp using sterile syringes with anticoagulant buffer $(450 \mathrm{mmol} / \mathrm{L} \mathrm{NaCl}$, $10 \mathrm{mmol} / \mathrm{L} \mathrm{KCl}, 10 \mathrm{mmol} / \mathrm{L}$ EDTA, $100 \mathrm{mmol} / \mathrm{L}$ HEPES, $\mathrm{pH} 7.45)$, and then the hemocytes were collected after centrifugation at $800 \mathrm{~g}$ for $6 \mathrm{~min}$ at $4{ }^{\circ} \mathrm{C}$. The other tissues were dissected with scissors and forceps from at least three shrimp, and placed on ice for RNA or protein extraction. The total RNAs from hemocytes and other organs (heart, hepatopancreas, gills, stomach and intestine) of the challenged and control shrimp were extracted using Unizol reagent (Biostar, Shanghai, China). cDNA was synthesized with a pair of primers (SMART F and Oligo anchor R) using a Revert Aid First Strand cDNA synthesis kit (Fermentas, Burlington, Canada).

Before challenge experiments, we randomly took three shrimp to detect if the shrimp are WSSV-free samples using RNA from hemocytes and intestines to analyze the expression of $V p 28 \mathrm{mRNA}$ with specific primers $\mathrm{Vp} 28 \mathrm{~F}$ and Vp28R by qPCR. The PCR was performed with the following procedures: $95^{\circ} \mathrm{C}$ for $10 \mathrm{~min} ; 40$ cycles at $95{ }^{\circ} \mathrm{C}$ for $10 \mathrm{~s}$ and $60{ }^{\circ} \mathrm{C}$ for $50 \mathrm{~s}$; and then a melting period from 65 to $95{ }^{\circ} \mathrm{C}$. The data obtained were analyzed using the cycle threshold $\left(2^{-\Delta \Delta \mathrm{CT}}\right)$ method. $\beta$-Actin was used as the internal control gene.

\section{Phylogenetic analysis}

The full-length coding sequences of MjALFs were obtained from hemocyte-, intestine-, and hepatopancreas-transcriptomic sequencing of $M$. japonicus in the laboratory. The ALF full-length sequences of other shrimp were collected from GenBank databases. Neighbor-joining analysis was conducted via MEGA 7, and bootstrap sampling was reiterated 500 times (Kumar et al. 2016).

\section{Semi-quantitative RT-PCR and quantitative real-time (qPCR) analysis}

The tissue distribution of MjALF-D2 in different tissues of non-injected shrimp were analyzed via RT-PCR with specific primers MjAlf RTF and RTR. $\beta$-actin was amplified as an internal control, with a pair of primers $\beta$-actin $\mathrm{F}$ and $\beta$-actin $\mathrm{R}$.

qPCR was performed to analyze the transcriptional profiles of MjAlf-D2 at different timepoints after WSSV challenge. The profile of the PCR was $95^{\circ} \mathrm{C}$ for $10 \mathrm{~min}, 40$ cycles of $95{ }^{\circ} \mathrm{C}$ for $15 \mathrm{~s}, 60^{\circ} \mathrm{C}$ for $60 \mathrm{~s}$, and read at $75^{\circ} \mathrm{C}$ for $2 \mathrm{~s}$; melting curve analysis was performed from 68 to $95^{\circ} \mathrm{C}$. The assay was repeated three times, and data were calculated using the $2^{-\Delta \Delta C t}$ method. Significant differences were analyzed using Student's $t$ test. The figures were created on Graphpad Prism 5 software.

\section{Expression and purification of four recombinant ALFs}

The mature peptide sequences of four MjALFs, including MjALF-C2, MjALF-D1, MjALF-D2, and MjALF-E1, were amplified with different primers (Table 1) via RTPCR; cloned into pET-32a (+); and transformed into E. coli Rosetta cells. The recombinant proteins were purified by affinity chromatography using High-affinity Ni-IDA Resin (Genscript, Nanjing, China). The parent vector pET-32a (+) was used to express the TRX-tag protein in the Rossetta cells. The control protein (TRX) was also purified using the same methods described above with High-affinity Ni-IDA Resin (Genscript, Nanjing, China).

\section{Antivirus activity analysis of recombinant ALFs}

WSSV inoculation was prepared following the previous method with a few modifications (Yang et al. 2016b). The gills (1 g) of WSSV-infected crayfish Procambarus clarkii were homogenized in $10 \mathrm{ml}$ PBS (140 mmol/L $\mathrm{aCl}$, 
Table 1 Primers used in the study

\begin{tabular}{lll}
\hline Primer & Sequence (5'-3') & Direction \\
\hline$\beta$-actinF & AGTAGCCGCCCTGGTTGTAGAC & Forward \\
$\beta$-actinR & TTCTCCATGTCGTCCCAGT & Reverse \\
MjALF-D2RNAi-F & GCGTAATACGACTCACTATAGGAGCACCTACAGTCATCAC & Forward \\
MjALF-D2RNAi-R & GCGTAATACGACTCACTATAGGAATCTCAAAGTTATTCTA & Reverse \\
Gfp RNAi-F & TAATACGACTCACTATAGGGGGGTGGTCCCAATTCTCGTGGAAC & Forward \\
Gfp RNAi-R & TAATACGACTCACTATAGGGCTTGTACAGCTCGTCCATGC & Reverse \\
MjAlf-D2RT-F & CGCAGGCTTATGGAGGAC & Forward \\
MjAlf-D2RT-R & AGGTGACAGTGCCGAGGA & Reverse \\
MjAlf-C2EXF & TACTCAGAATTCCAGGGGTGGGAGGCACTCGTGCCA & Forward \\
MjAlf-C2EXR & TACTCACTCGAG TTACTGATTTAACCAAGCCT & Reverse \\
MjAlf-D1EXF & TACTCAGAATTCCAAATATGGGAGACGCTGAT & Forward \\
MjAlf-D1EXR & TACTCACTCGAGTTACTTGTTGAGCCACGCCT & Reverse \\
MjAlf-D2EXF & TACTCAGAATTCCAGGGACTAAAGGACTTTTTAT & Forward \\
MjAlf-D2EXR & TACTCACTCGAGCTACACGATATATGGTTTTG & Reverse \\
MjAlf-E2EXF & TACTCAGAATTCATGATGACGTCACCCAATCC & Forward \\
MjAlf-E2EXR & TACTCACTCGAGTTACAGCCACTCTGCCGCTT & Reverse \\
Vp28F & AGCTCCAACACCTCCTCCTTCA & Forward \\
Vp28R & TTACTCGGTCTCAGTGCCAGA & Reverse \\
Ie1F & GACTCTACAAATCTCTTTGCCA & Forward \\
Ie1R & CTACCTTTGCACCAATTGCTAG & Reverse \\
\hline
\end{tabular}

$2.7 \mathrm{mmol} / \mathrm{L} \mathrm{KCl}, 10 \mathrm{mmol} / \mathrm{L} \mathrm{Na}_{2} \mathrm{HPO}_{4}$, and $1.8 \mathrm{mmol} / \mathrm{L}$ $\mathrm{KH}_{2} \mathrm{PO}_{4} ; \mathrm{pH} 7.4$ ), and then centrifuged at $5000 \mathrm{~g}$ for $10 \mathrm{~min}$ at $4{ }^{\circ} \mathrm{C}$. The supernatant was filtered through a $0.45 \mu \mathrm{m}$ filter (Jinlong, Tianjin, China). The viral titer was determined via qPCR on the basis of a previous report (Wang et al. 2014b). M. japonicus were divided into three groups, and the shrimp in each group was injected with PBS, TRX, or MjALFs $(30 \mu \mathrm{g})$. One hour later, the shrimp was challenged with WSSV $\left(2.5 \times 10^{5}\right.$ copies $)$. Genomic DNA, mRNA, and proteins were extracted from the gills of WSSV-infected and control shrimp at 36 and $48 \mathrm{~h}$ post WSSV injection. The genomic DNA was extracted using the MagEtractor Genomic DNA purification kit (Toyobo, Shanghai, China), and WSSV replication was analyzed using qPCR, with Vp28, a WSSV envelope protein, as an indicator.

\section{RNA interference assay}

The dsRNAs of MjALF-D2 or green fluorescent protein (GFP) were synthesized using the DNA template amplified with specific primers $M j$ Alf-D2RNAiF, RNAiR, GfpF, and GfpR linked to the T7 promoter (Table 1) by T7 RNA polymerase (Fermentas, Thermo Fisher Scientific, USA). The shrimp were randomly divided into two groups. Each shrimp in the two groups was injected with $30 \mu \mathrm{g}$ of $d s M j A l f-D 2$ or $d s G f p$ two times every $24 \mathrm{~h}$. Total RNA was extracted from the gills of the shrimp at $48 \mathrm{~h}$ post second injection using TRIzol (TransGen, Beijing, China) to detect the RNAi efficiency. Then, WSSV $\left(2.5 \times 10^{5}\right.$ copies $)$ was injected into the
MjAlf-D2-silenced shrimp at $48 \mathrm{~h}$ post second $d s R N A$ injection. Genomic DNA, mRNA, and proteins were extracted from the gills of the shrimp at 36 and $48 \mathrm{~h}$ post WSSV injection using the method described above for the detection of WSSV replication.

\section{Western blotting}

Western blotting was used to detect WSSV replication by using VP28 antibody. The gills of MjAlf-D2-RNAi shrimp were homogenized in the buffer $(50 \mathrm{mmol} / \mathrm{L}$ Tris $-\mathrm{HCl}$, $150 \mathrm{mmol} / \mathrm{L} \mathrm{NaCl}, 1 \mathrm{mmol} / \mathrm{L} \mathrm{EDTA}$, and $1 \mathrm{mmol} / \mathrm{L}$ phenylmethylsulfonyl fluoride; $\mathrm{pH} 7.5)$, and the homogenate was centrifuged at $10,000 \mathrm{~g}$ for $10 \mathrm{~min}$ at $4{ }^{\circ} \mathrm{C}$. The proteins of the sample were separated by $12.5 \%$ SDS-polyacrylamide gel electrophoresis and transferred onto nitrocellulose membranes. The membranes were blocked with $3 \%$ nonfat milk in TBS $(10 \mathrm{mmol} / \mathrm{L}$ Tris- $\mathrm{HCl}$ and $150 \mathrm{mmol} / \mathrm{L} \mathrm{NaCl}$; pH 7.5) for $1 \mathrm{~h}$, and incubated for $2 \mathrm{~h}$ with 1/100 diluted antiserum against VP28 in TBS with $3 \%$ nonfat milk. Then, after washing to remove the free, nonspecifically binding antiserum, horseradish peroxidase-conjugated goat anti-rabbit IgG (1:10,000 dilution in TBS) was added and incubated for $2 \mathrm{~h}$. The membrane was put in the reaction system $(10 \mathrm{ml}$ of TBS with $45 \mu \mathrm{l}$ of NBT and $35 \mu \mathrm{l}$ of BCIP) in the dark for $5 \mathrm{~min}$ to visualize the signal. The antibody against the VP28 and $\beta$-actin used in Western blotting was prepared in the laboratory. The bands on the membrane of western blot 
of three independent repeats were digitalized by scanning using ImageJ software.

\section{Statistical analysis}

Data are presented as mean $\pm \operatorname{SD}(n=3)$. Statistical evaluation of significant differences between experimental groups was conducted using Student's $t$ test. The $P$ values are defined as follows: $* P<0.05$; $* * P<0.01 ; * * * P<0.001$.

Acknowledgements This research is supported by grants from the National Natural Science Foundation of China (grant nos. 31630084 and 31930112), and the National Key Research and Development Program of China (grant no. 2018YFD0900502).

Author contributions HSJ and JXW conceived and designed the study. HSJ and LXL performed the experiments, HSJ, LXL and JXW contributed to data analysis and interpretation. HSJ and JXW wrote the manuscript. All authors edited and approved the final manuscript.

\section{Declarations}

Conflict of interest All the authors declare that there are no conflicts of interest.

Animal and human rights statement The shrimp used in our study are consumed frequently by the wider community, so the approval according to the regulations on the use of shrimp is unnecessary.

Open Access This article is licensed under a Creative Commons Attribution 4.0 International License, which permits use, sharing, adaptation, distribution and reproduction in any medium or format, as long as you give appropriate credit to the original author(s) and the source, provide a link to the Creative Commons licence, and indicate if changes were made. The images or other third party material in this article are included in the article's Creative Commons licence, unless indicated otherwise in a credit line to the material. If material is not included in the article's Creative Commons licence and your intended use is not permitted by statutory regulation or exceeds the permitted use, you will need to obtain permission directly from the copyright holder. To view a copy of this licence, visit http://creativecommons.org/licenses/by/4.0/.

\section{References}

Afsal VV, Antony SP, Chaithanya ER, Singh IS, Philip R (2012) Two isoforms of anti-lipopolysaccharide factors identified and characterized from the hemocytes of portunid crabs, Portunus pelagicus and Scylla tranquebarica. Mol Immunol 52:258-263

Bi WJ, Li DX, Xu YH, Xu S, Li J, Zhao XF, Wang JX (2015) Scavenger receptor B protects shrimp from bacteria by enhancing phagocytosis and regulating expression of antimicrobial peptides. Dev Comp Immunol 51:10-21

de la Vega E, O’Leary NA, Shockey JE, Robalino J, Payne C, Browdy CL, Warr GW, Gross PS (2008) Anti-lipopolysaccharide factor in Litopenaeus vannamei (LvALF): a broad spectrum antimicrobial peptide essential for shrimp immunity against bacterial and fungal infection. Mol Immunol 45:1916-1925
Hanson MA, Dostalova A, Ceroni C, Poidevin M, Kondo S, Lemaitre B (2019) Synergy and remarkable specificity of antimicrobial peptides in vivo using a systematic knockout approach. Elife 8:e44341

Jenssen H, Hamill P, Hancock RE (2006) Peptide antimicrobial agents. Clin Microbiol Rev 19:491-511

Jiang HS, Sun C, Wang T, Zhao XF, Wang JX (2013) A single whey acidic protein domain containing protein (SWD) inhibits bacteria invasion and dissemination in shrimp Marsupenaeus japonicus. Fish Shellfish Immunol 35:310-318

Jiang HS, Zhang Q, Zhao YR, Jia WM, Zhao XF, Wang JX (2015) A new group of anti-lipopolysaccharide factors from Marsupenaeus japonicus functions in antibacterial response. Dev Comp Immunol 48:33-42

Jiravanichpaisal P, Lee SY, Kim YA, Andren T, Soderhall I (2007) Antibacterial peptides in hemocytes and hematopoietic tissue from freshwater crayfish Pacifastacus leniusculus: characterization and expression pattern. Dev Comp Immunol 31:441-455

Kumar S, Stecher G, Tamura K (2016) MEGA7: molecular evolutionary genetics analysis version 7.0 for bigger datasets. Mol Biol Evol 33:1870-1874

Li S, Guo S, Li F, Xiang J (2014) Characterization and function analysis of an anti-lipopolysaccharide factor (ALF) from the Chinese shrimp Fenneropenaeus chinensis. Dev Comp Immunol 46:349-355

Liu F, Liu Y, Li F, Dong B, Xiang J (2005) Molecular cloning and expression profile of putative antilipopolysaccharide factor in Chinese shrimp (Fenneropenaeus chinensis). Mar Biotechnol (NY) 7:600-608

Liu H, Jiravanichpaisal P, Soderhall I, Cerenius L, Soderhall K (2006) Antilipopolysaccharide factor interferes with white spot syndrome virus replication in vitro and in vivo in the crayfish Pacifastacus leniusculus. J Virol 80:10365-10371

Matos GM, Schmitt P, Barreto C, Farias ND, Toledo-Silva G, Guzman F, Destoumieux-Garzon D, Perazzolo LM, Rosa RD (2018) Massive gene expansion and sequence diversification is associated with diverse tissue distribution, regulation and antimicrobial properties of anti-lipopolysaccharide factors in shrimp. Mar Drugs $16: 381$

Methatham T, Boonchuen P, Jaree P, Tassanakajon A, Somboonwiwat K (2017) Antiviral action of the antimicrobial peptide ALFPm3 from Penaeus monodon against white spot syndrome virus. Dev Comp Immunol 69:23-32

Mookherjee N, Anderson MA, Haagsman HP, Davidson DJ (2020) Antimicrobial host defence peptides: functions and clinical potential. Nat Rev Drug Discov 19:311-332

Morita T, Ohtsubo S, Nakamura T, Tanaka S, Iwanaga S, Ohashi K, Niwa M (1985) Isolation and biological-activities of Limulus anticoagulant (Anti-Lps Factor) which interacts with lipopolysaccharide (LPS). J Biochem 97:1611-1620

Ng TH, Chang SH, Wu MH, Wang HC (2013) Shrimp hemocytes release extracellular traps that kill bacteria. Dev Comp Immunol 41:644-651

Niu GJ, Wang S, Xu JD, Yang MC, Sun JJ, He ZH, Zhao XF, Wang JX (2019) The polymeric immunoglobulin receptor-like protein from Marsupenaeus japonicus is a receptor for white spot syndrome virus infection. PLoS Pathog 15:e1007558

Ohashi K, Niwa M, Nakamura T, Morita T, Iwanaga S (1984) Anti-LPS factor in the horseshoe crab, Tachypleus tridentatus: Its hemolytic activity on the red blood cell sensitized with lipopolysaccharide. FEBS Lett 176:207-210

Peng SE, Lo CF, Lin SC, Chen LL, Chang YS, Liu KF, Su MS, Ko GH (2001) Performance of WSSV-infected and WSSV-negative Penaeus monodon postlarvae in culture ponds. Dis Aquat Organ 46:165-172

Ren Q, Zhang Z, Li XC, Jie D, Hui KM, Zhang CY, Wang W (2012) Three different anti-lipopolysaccharide factors identified from 
giant freshwater prawn, Macrobrachium rosenbergii. Fish Shellfish Immunol 33:766-774

Rosa RD, Vergnes A, de Lorgeril J, Goncalves P, Perazzolo LM, Sauné L, Romestand B, Fievet J, Gueguen Y, Bachère E, DestoumieuxGarzón D (2013) Functional divergence in shrimp anti-lipopolysaccharide factors (ALFs): from recognition of cell wall components to antimicrobial activity. PLoS ONE 8:e67937

Schmitt P, Rosa RD, Destoumieux-Garzon D (2016) An intimate link between antimicrobial peptide sequence diversity and binding to essential components of bacterial membranes. Biochim Biophys Acta 1858:958-970

Sun C, Xu WT, Zhang HW, Dong LP, Zhang T, Zhao XF, Wang JX (2011) An anti-lipopolysaccharide factor from red swamp crayfish, Procambarus clarkii, exhibited antimicrobial activities in vitro and in vivo. Fish Shellfish Immunol 30:295-303

Supungul P, Jaree P, Somboonwiwat K, Junprung W, Proespraiwong P, Mavichak R, Tassanakajon A (2017) A potential application of shrimp antilipopolysaccharide factor in disease control in aquaculture. Aquaculture Res 48:809-821

Suraprasit S, Methatham T, Jaree P, Phiwsaiya K, Senapin S, Hirono I, Lo CF, Tassanakajon A, Somboonwiwat K (2014) Anti-lipopolysaccharide factor isoform 3 from Penaeus monodon (ALFPm3) exhibits antiviral activity by interacting with WSSV structural proteins. Antiviral Res 110:142-150

Tanaka S, Nakamura T, Morita T, Iwanaga S (1982) Limulus anti-LPS factor - an anticoagulant which inhibits the endotoxin-mediated activation of Limulus coagulation system. Biochem Biophys Res Commun 105:717-723

Tassanakajon A, Amparyup P, Somboonwiwat K, Supungul P (2011) Cationic antimicrobial peptides in penaeid shrimp. Mar Biotechnol (NY) 13:639-657
Tassanakajon A, Rimphanitchayakit V, Visetnan S, Amparyup P, Somboonwiwat K, Charoensapsri W, Tang S (2018) Shrimp humoral responses against pathogens: antimicrobial peptides and melanization. Dev Comp Immunol 80:81-93

Wang XW, Wang JX (2013) Pattern recognition receptors acting in innate immune system of shrimp against pathogen infections. Fish Shellfish Immunol 34:981-989

Wang PH, Huang T, Zhang X, He JG (2014a) Antiviral defense in shrimp: from innate immunity to viral infection. Antiviral Res 108:129-141

Wang XW, Xu YH, Xu JD, Zhao XF, Wang JX (2014b) Collaboration between a soluble C-type lectin and calreticulin facilitates white spot syndrome virus infection in shrimp. J Immunol 193:2106-2117

Yang Y, Boze H, Chemardin P, Padilla A, Moulin G, Tassanakajon A, Pugniere M, Roquet F, Destoumieux-Garzón D, Gueguen Y, Bachère E, Aumelas A (2009) NMR structure of rALF-Pm3, an anti-lipopolysaccharide factor from shrimp: model of the possible lipid A-binding site. Biopolymers 91:207-220

Yang HT, Yang MC, Sun JJ, Shi XZ, Zhao XF, Wang JX (2016a) Dual oxidases participate in the regulation of intestinal microbiotic homeostasis in the kuruma shrimp Marsupenaeus japonicus. Dev Comp Immunol 59:153-163

Yang MC, Shi XZ, Yang HT, Sun JJ, Xu L, Wang XW, Zhao XF, Wang JX (2016) Scavenger receptor C mediates phagocytosis of white spot syndrome virus and restricts virus proliferation in shrimp. PLoS Pathog 12:e1006127

Zheng SC, Xu JY, Liu HP (2019) Cellular entry of white spot syndrome virus and antiviral immunity mediated by cellular receptors in crustaceans. Fish Shellfish Immunol 93:580-588 